\section{Revolución digital en salud: ¿están los pacientes con accidente cerebrovascular preparados?}

\author{
PAULINA SÁEZ GALLARDO ${ }^{1, a}$, TERESITA RISOPATRÓN RIESCO ${ }^{1}$, \\ RODRIGO GUERRERO T. ${ }^{1,2}$
}

\section{Digital revolution in healthcare: are stroke patients ready?}

Background: The digital revolution is creating opportunities and challenges in the field of medicine and the Digital Hospital could be a benefit for patients, if they are able to use the Internet. Aim: Identify the barriers that limit Internet use among stroke patients treated in a public hospital in Santiago. Material and Methods: Sociodemographic, clinical and functional variables that could influence the use of the Internet were collected in 107 patients with stroke. Results: Seventy five percent of patients had access to the Internet, but only 29\% knew how to use it before their hospitalization. Seventy five percent were older adults, 74\% had a low level of education, 78\% had an income below the minimum Chilean salary and $56 \%$ were discharged with a severe disability (modified Rankin Scale 4 or 5). There was a significant relationship between Internet use and the level of education, income and age. Conclusions: Income levels, age, schooling and stroke related disability limit Internet use in this population.

(Rev Med Chile 2020; 148: 787-791)

Key words: Digital Divide; Internet; Stroke; Telemedicine.

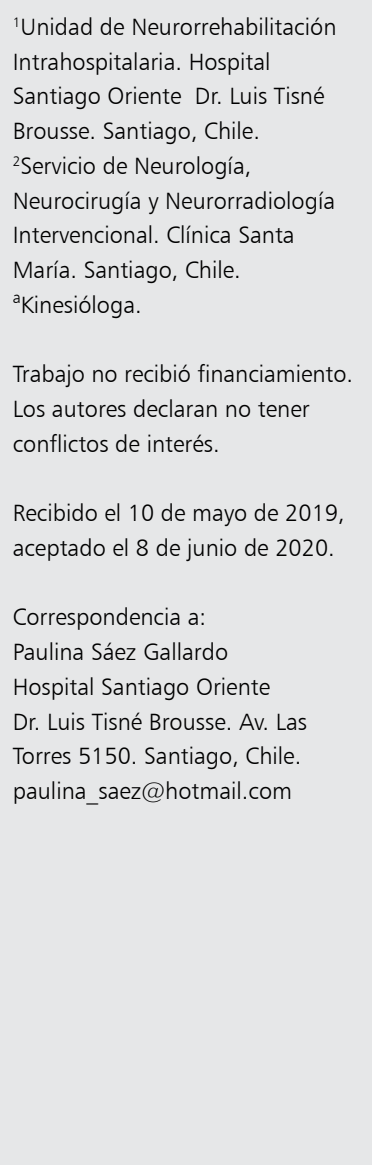

'Unidad de Neurorrehabilitación Intrahospitalaria. Hospital Santiago Oriente Dr. Luis Tisné Usse. Santiago, Chile. Intervencional. Clínica Santa

María. Santiago, Chile.

Trabajo no recibió financiamiento Los autores declaran no tener

Recibido el 10 de mayo de 2019

Correspondencia a:

Paulina Sáez Gallardo Hospital Santiago Oriente Dr. Luis Tisné Brousse. Av. Las Torres 5150. Santiago, Chile. paulina_saez@hotmail.com
$\mathrm{L}$ a revolución digital está creando oportunidades y desafíos sin precedentes en el campo de la medicina y se ha convertido en un gran aliado a la hora de crear avances que podrían llevar a los establecimientos de salud, no solo a tener una mayor eficiencia y reducir costos, sino que incluso a mejorar la calidad de vida de los profesionales de la salud y sus pacientes, permitiendo un acceso rápido a información decisiva para diagnosticar y tratar. Esto puede significar un enorme beneficio, pero ¿estamos preparados?

Actualmente, el Ministerio de Salud se encuentra desarrollando políticas que involucran la utilización de tecnologías de la información y comunicación (TIC), una de las más destacadas es el Hospital Digital. "Surge porque nos hemos propuesto cambiar la cara de la salud pública [...] para que todos perciban que el desarrollo también ha llegado a la salud de manera inclusiva [...]" señaló el ex Ministro de Salud Emilio Santelices en una columna de opinión ${ }^{1}$.

Dentro de sus componentes, el Hospital Digital contempla una plataforma web y una aplicación móvil (APP), que pondrán a disposición de la población una serie de servicios, desde la consulta de precios de medicamentos y disponibilidad de horas de atención, hasta la atención médica remota.

Uno de los desafíos que supone la implementación de este proyecto es romper la brecha digital existente, tanto de acceso a internet, como de las habilidades y competencias necesarias para usarlo. Actualmente, diversos estudios proveen evidencia sobre la influencia de las características sociodemográficas de la población en el acceso y uso de internet $^{2-8}$. En Chile, el estudio realizado por la Fundación País Digital identifica 4 factores que 
afectan el uso de internet: el nivel de ingresos, la edad, la escolaridad y la ruralidad ${ }^{2}$. Por otra parte, esta brecha puede ser aun mayor en personas con discapacidad, ya que alteraciones sensoriales, cognitivas y motoras también podrían afectar el acceso y uso de las TIC ${ }^{9-11}$.

El objetivo del presente artículo es identificar los factores que podrían influir en el uso de internet en las personas con diagnóstico de accidente cerebrovascular (ACV) ingresadas en la Unidad de Neurorrehabilitación Intrahospitalaria del Hospital Santiago Oriente - Dr. Luis Tisné Brousse (HSO).

\section{Materiales y Métodos}

Se realizó un estudio retrospectivo y descriptivo para caracterizar a los pacientes con diagnóstico de ACV atendidos en la Unidad de Neurorrehabilitación Intrahospitalaria del HSO, en Santiago de Chile, durante el año 2018. Para ello se revisaron las fichas clínicas y las evaluaciones realizadas por el equipo de neurorrehabilitación al ingreso y al alta hospitalaria. Por protocolo, a todos los pacientes con ACV que ingresan a la unidad y a los familiares que viven con ellos, se les aplica una encuesta que incluye información acerca del acceso y uso de internet fijo o móvil. Se definió "uso de internet" como la habilidad operacional de entrar a una página web o de bajar una APP.

Se recolectaron las variables sociodemográficas, clínicas y funcionales que podrían influir en el uso de internet según la literatura ${ }^{2,10}$ (edad, género, tramo Fonasa, diagnóstico vascular, grado de discapacidad, deterioro cognitivo y déficits sensoriales). El grado de discapacidad al alta fue evaluado con la escala de Rankin modificada $(\mathrm{mRS})^{12}$. El deterioro cognitivo fue evaluado con la prueba de memoria, fluidez y orientación (MEFO) ${ }^{13}$, se consideró deterioro cognitivo un puntaje $\leq 8$. Los déficits sensoriales que se consignaron fueron el visual y el auditivo ${ }^{10}$, considerando como déficit auditivo aquel que no permite mantener una conversación fluida y visual aquel que restringe la capacidad de leer, ya sea por problemas en el campo o la agudeza visual. Se utilizó el tramo Fonasa como aproximación a la situación económica de los pacientes.

Los datos se analizaron mediante estadística descriptiva y se aplicó la prueba de chi-cuadrado para determinar la relación entre el uso de internet y las variables sociodemográficas de la población. Para ello se utilizó el programa estadístico Jamovi. El nivel de significancia fue definido como $\mathrm{p}<0,05$.

El trabajo fue aprobado por el Comité de Ética del Servicio de Salud Metropolitano Oriente y del HSO.

\section{Resultados}

El estudio se llevó a cabo con una base de datos de 113 pacientes ingresados entre mayo y diciembre de 2018, con diagnóstico de ACV confirmado por neuroimagen y neurólogo de la unidad. De estos pacientes, 6 fallecieron durante la hospitalización, por lo tanto, se analizaron 107 fichas clínicas. Las características socioeconómicas, demográficas, clínicas y funcionales de la población se describen en la Tabla 1.

La media de edad de los pacientes evaluados fue de 70,8 años (DS: 13,4). El 5,6\% de la población era analfabeta o no escolarizada y $68,2 \%$ tenía escolaridad incompleta. El 10,3\% de los pacientes pertenecía al tramo Fonasa A y 67,3\% al B, lo que significa que $77,6 \%$ percibía un ingreso imponible mensual menor o igual al sueldo mínimo. El 74,8\% de los pacientes tenía acceso a internet, pero solo $28,8 \%$ de estos lo sabía utilizar previo a su hospitalización. El 56,2\% de los pacientes fue dado de alta del HSO con una discapacidad moderadamente grave a grave (mRS 4 o 5 ).

Se encontró una relación estadísticamente significativa entre el uso de internet y las variables sociodemográficas de edad, escolaridad y nivel de ingresos; siendo más probable que no usen internet los adultos mayores, las personas con escolaridad incompleta y aquellas con un nivel de ingresos menor o igual al sueldo mínimo (Tabla 2). Esta probabilidad aumenta significativamente $(\mathrm{p}<0,001)$ en los adultos mayores con escolaridad incompleta (OR 17,7 IC 95\% 4,8-65,2).

\section{Discusión}

El presente estudio muestra que, si bien un alto porcentaje de los pacientes evaluados tenía acceso a internet $(74,8 \%)$, solo $28,8 \%$ de ellos lo sabía usar. Es posible que esto se deba a las características sociodemográficas de la población, 
Tabla 1. Características sociodemográficas, clínicas y funcionales de la población estudiada

\begin{tabular}{|c|c|}
\hline Características & n (\%) \\
\hline Adultos mayores ( $\geq 65$ años) & $80(74,8)$ \\
\hline Género femenino & $62(57,9)$ \\
\hline $\begin{array}{l}\text { Escolaridad } \\
\text { No escolarizado o analfabeto } \\
\text { Básica incompleta } \\
\text { Básica completa } \\
\text { Media incompleta } \\
\text { Media completa }\end{array}$ & $\begin{array}{r}6(5,6) \\
43(40,2) \\
18(16,8) \\
12(11,2) \\
28(26,2)\end{array}$ \\
\hline $\begin{array}{l}\text { Tramo Fonasa } \\
\text { A } \\
\text { B } \\
\text { C } \\
\text { D }\end{array}$ & $\begin{array}{r}11(10,3) \\
72(67,3) \\
9 \quad(8,4) \\
15(14,0)\end{array}$ \\
\hline $\begin{array}{l}\text { Pacientes con acceso a internet } \\
\text { Sí }\end{array}$ & $80(74,8)$ \\
\hline $\begin{array}{l}\text { Pacientes que saben usar internet } \\
\text { Sí }\end{array}$ & $23(28,8)$ \\
\hline $\begin{array}{l}\text { Diagnóstico } \\
\text { ACV isquémico } \\
\text { ACV hemorrágico }\end{array}$ & $\begin{array}{l}85(79,4) \\
22(20,6)\end{array}$ \\
\hline $\begin{array}{l}\text { Territorio afectado } \\
\text { Hemisferio cerebral izquierdo } \\
\text { Hemisferio cerebral derecho } \\
\text { Ambos hemisferios cerebrales } \\
\text { Fosa posterior }\end{array}$ & $\begin{array}{r}50(46,7) \\
40(37,4) \\
5 \quad(4,7) \\
12(11,2)\end{array}$ \\
\hline $\begin{array}{l}\text { Oxfordshire } \\
\text { PACI } \\
\text { LACI } \\
\text { POCI } \\
\text { TACI }\end{array}$ & $\begin{array}{r}29(27,1) \\
27(25,2) \\
21(19,6) \\
8(7,5)\end{array}$ \\
\hline $\begin{array}{l}\text { TOAST } \\
\text { Oclusión de pequeño vaso } \\
\text { Ateroesclerosis de grandes vasos } \\
\text { Cardioembólico } \\
\text { Otras etiologías } \\
\text { Etiología indeterminada }\end{array}$ & $\begin{array}{r}28(26,2) \\
24(22,4) \\
8 \quad(7,5) \\
3 \quad(2,8) \\
22(20,6)\end{array}$ \\
\hline $\begin{array}{l}\text { Déficit sensorial } \\
\text { Visual } \\
\text { Auditivo } \\
\text { Mixto }\end{array}$ & $\begin{array}{r}30(28,0) \\
11(10,3) \\
1(0,9)\end{array}$ \\
\hline $\begin{array}{l}\text { Deterioro cognitivo } \\
\text { MEFO } \leq 8 \\
\text { Diagnóstico previo } \\
\text { Afasia (MEFO no aplicable) }\end{array}$ & $\begin{array}{r}41(38,3) \\
9(8,4) \\
14(13,1)\end{array}$ \\
\hline $\begin{array}{l}\text { Grado de discapacidad al alta (mRS) } \\
0 \\
1 \\
2 \\
3 \\
4 \\
5\end{array}$ & $\begin{aligned} & 1(0,9) \\
& 14(13,1) \\
& 14(14,9) \\
& 16(14,9) \\
& 48(45,0) \\
& 12(11,2)\end{aligned}$ \\
\hline
\end{tabular}

PACl: Partial Anterior Circulation Infarcts, LACl: Lacunar Infarcts, POCI: Posterior Circulation Infarcts, TACl: Total Anterior Circulation Infarcts. TOAST: Trial of Org 10172 in Acute Stroke Treatment. donde la edad, la escolaridad y el nivel de ingresos tienen un impacto significativo en la probabilidad de usar o no internet, apoyando la evidencia disponible $2,3,5,8,9,15,16$.

A lo anterior se agregan dificultades vinculadas a la condición de salud de la población estudiada $^{9,10,11,17}$. El 39,2\% presentaba discapacidad sensorial y $59,8 \%$, deterioro cognitivo. Además, $56,2 \%$ fue dado de alta con un mRS 4 o 5 , es decir, se encontraba postrado o requería asistencia para realizar la mayoría de sus actividades de la vida diaria. A partir de esta aproximación funcional, se puede inferir que un alto porcentaje de pacientes no podría utilizar internet sin ayuda de otra persona o de tecnologías asistivas. En estos casos, la conexión digital podría significar un avance para establecer contacto con los cuidadores.

En suma, las potenciales barreras para el uso de internet identificadas en este estudio son: el nivel de ingresos, la edad, la escolaridad, el deterioro cognitivo, la discapacidad y los déficits sensoriales (Figura 1). Si bien estos pacientes son una pequeña muestra de los usuarios que se beneficiarían del Hospital Digital, el resto de la población no está exenta de enfrentarse a alguna de ellas. Por lo tanto, al momento de diseñar la plataforma virtual dirigida a los pacientes, se debe tener en cuenta este conjunto de barreras, a fin de lograr un diseño inclusivo, que sea de uso sencillo e intuitivo, fácil de aprender, fácil de utilizar y fácil de recordar. Además, de existir espacios habilitados para el acceso al Hospital Digital, como los centros de Salud Familiar, sería importante considerar la existencia de tecnologías asistivas o funcionarios que faciliten el uso de esta herramienta a quienes no tienen la capacidad física o cognitiva para hacerlo sin ayuda. Por último, los estudios demuestran que los factores que más impactan en el uso de internet son la edad y el nivel educacional ${ }^{2,15,16,18,19}$. Por tanto, para potenciar el efecto de esta iniciativa habría que ampliar los esfuerzos que ya se realizan a nivel país, hacia superar la brecha educacional y mejorar las competencias digitales de la población por medio de acciones de alfabetización digital ${ }^{17,20}$.

Las principales limitaciones del presente estudio guardan relación con el diseño del estudio y su consecuente sesgo de memoria. Además, no se cuenta con la información de "uso de internet" posterior al alta hospitalaria, por ende, la influencia de las variables clínicas sobre este es desconocida. Por último, sería deseable contar 
Tabla 2. Factores que influyen en el no uso de internet. Análisis estadístico de las variables sociodemográficas según la prueba de chi-cuadrado

\begin{tabular}{|lrc|}
\hline Variable & OR (IC 95\%) & p \\
\hline Edad $\geq 65$ & $11,3(3,9-32,3)$ & $<, 001$ \\
Género Femenino & $1,4(0,5-3,4)$ & 0,527 \\
Escolaridad incompleta $<12$ años & $7,8(2,8-21,5)$ & $<0,001$ \\
Nivel de ingresos menor o igual al sueldo mínimo* & $2,9(1,1-8,1)$ & 0,030 \\
\hline
\end{tabular}

*Año 2018.

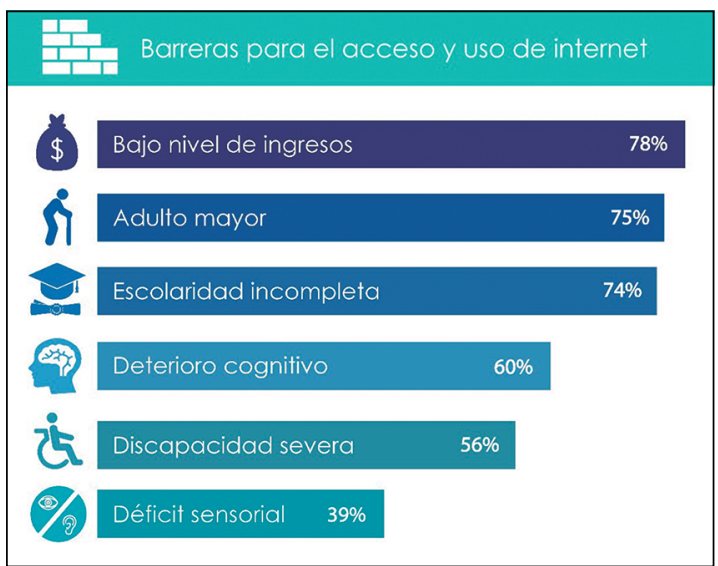

Figura 1. Infografía acerca de las posibles barreras para el acceso y uso de internet presentes en la población con ACV del HSO.

con información más específica sobre el nivel de ingresos y una evaluación objetiva de los déficits sensoriales.

\section{Conclusión}

Los factores que más interfieren en el uso de internet en la población con ACV del HSO previo a su hospitalización son: el nivel de ingresos, la edad y la escolaridad, sumando, además, la barrera potencial de la discapacidad producto del ACV. Por lo tanto, si se quiere un impacto global y actual del Hospital Digital, en lo que respecta a trámites realizados por el paciente a través de internet, es necesaria una planificación inclusiva. De lo contrario, el beneficio de esta revolución digital en salud lo recibirá una parte de los pacientes o bien, los usuarios del futuro.

\section{Referencias}

1. Santelices E. Ministro de Salud. Por qué un Hospital Digital. El Mercurio, Economía y Negocios. Disponible en: www.economiaynegocios.cl [Consultado el 18 de febrero de 2019].

2. León R, Meza S. Brecha en el uso de Internet: una expresión de la exclusión social. Fundación País Digital. Santiago de Chile. 2018. Disponible en: www.paisdigital. org [Consultado el 14 de febrero de 2019].

3. Kruse C, Karem P, Shifflett K, Vegi L, Ravi K, Brooks M. Evaluating barriers to adopting telemedicine worldwide: A systematic review. J Telemed Telecare 2018; 24 (1): 4-12.

4. Van Deursen AJ, Van Dijk JA, Peters O. Rethinking Internet skills: The contribution of gender, age, education, Internet experience, and hours online to medium- and content-related Internet skills. Poetics 2011; 39 (2): 125144.

5. Van Deursen AJ, Van Dijk JA. Internet skill levels increase, but gaps widen: a longitudinal crosssectional analysis (2010-2013) among the Dutch population. Information, Communication and Society 2014; 18 (7): 782-97.

6. Kontos E, Blake KD, Chou WYS, Prestin A. Predictors of eHealth Usage: Insights on The Digital Divide From the Health Information National Trends Survey 2012. J Med Internet Res 2014; 16 (7): e172.

7. Ono H, Zavodny M. Digital inequality: A five country comparison using microdata. Soc Sci Res 2007; 36 (3): 1135-55.

8. Lera-López F, Gil Izquierdo M, Billón-Currás, M. El uso de Internet en España: Influencia de factores regionales y socio-demográficos. Investigaciones Regionales 2009; 16: 93-115.

9. Silva P, Matos A, Martínez-Pecino, R. E-inclusion: Beyond individual socio-demographic characteristics Plos One 2017; 12 (9): e0184545. 
10. Guenaga ML, Barbier A, Eguíluz A. La accesibilidad y las tecnologías en la información y la comunicación. TRANS. Revista de Traductología 2007; 11: 155-69.

11. Gell NM, Rosenberg DE, Demiris G, Lacroix AZ, Patel KV. Patterns of Technology Use Among Older Adults With and Without Disabilities. Gerontologist 2013; $1-11$.

12. Taylor-Rowan M, Wilson A, Dawson J, Quinn TJ. Functional Assessment for Acute Stroke Trials: Properties, Analysis and Application. Front Neurol 2018; 9: 191.

13. Delgado C, Guerrero S, Troncoso M, Araneda A, Slachevsky A, Behrens MI. Memoria, fluidez y orientación: prueba de cribado de deterioro cognitivo en 5 minutos. Neurología 2013; 28 (7): 400-7.

14. Van Deursen AJ, Van Dijk JA. Internet skills performance tests: are people ready for eHealth? J Med Internet Res 2011; 13 (2): e35.

15. Loges WE, Jung J-Y. Exploring the digital divide. Inter- net connectedness and age. Communication Res 2001; 28 (4): 536-562.

16. Niehaves B, Plattfaut R. Internet adoption by the elderly: employing IS technology acceptance theories for understanding the age-related digital divide. Eur J Inf Syst 2014; 23 (6): 708-26.

17. Morris A. E-literacy and the grey digital divide: a review with recommendations. J Information Literacy 2007; 1 (3): 13-28.

18. Friemel TN. The digital divide has grown old: Determinants of a digital divide among seniors. New Media and Society 2014; 1-19.

19. Ramón-Jerónimo MA, Peral-Peral B, Arenas-Gaitán J. Elderly Persons and Internet Use. Social Science Computer Review 2013; 31 (4): 389-403.

20. Miwa M, Nishina E, Kurosu M, Takahashi H, Yaginuma Y, Hirose Y, et al. Changing patterns of perceived ICT skill levels of elderly learners in a digital literacy training course. Libres 2017; 27 (1): 13-25. 